\title{
Moment Generating Function of the AoI in Multi-Source Systems with Computation-Intensive Status Updates
}

\author{
Mohammad Moltafet and Markus Leinonen \\ Centre for Wireless Communications - Radio Technologies \\ University of Oulu, Finland \\ e-mail: \{mohammad.moltafet, markus.leinonen\}@oulu.fi
}

\author{
Marian Codreanu \\ Department of Science and Technology \\ Linköping University, Sweden \\ e-mail: marian.codreanu@liu.se
}

\begin{abstract}
We consider a multi-source status update system in which status updates are transmitted as packets containing the measured value of the monitored process and a time stamp representing the time when the sample was generated. The packets of each source are generated according to the Poisson process and the packets are served according to an exponentially distributed service time. We assume that the received status update packets need further processing before being used (hence, computationintensive). This is mathematically modeled by introducing an additional server at the sink node. The sink server serves the packets according to an exponentially distributed service time. We introduce two packet management policies, namely, i) a preemptive policy and ii) a blocking policy and derive the moment generating function (MGF) of the AoI of each source under both policies. In the preemptive policy, a new arriving packet preempts any possible packet that is currently under service regardless of the packet's source index. In the blocking policy, when a server is busy at the arrival instant of a packet the arriving packet is blocked and cleared. We assume that the same preemptive/blocking policy is employed in both transmitter and sink servers. Numerical results are provided to assess the results.

Index Terms- Age of information (AoI), stochastic hybrid systems (SHS), computation-intensive status updates.
\end{abstract}

\section{INTRODUCTION}

Freshness of status information of various physical processes is a key performance enabler in many time-critical applications of wireless sensor networks (WSNs), e.g., surveillance in smart home systems, remote surgery, intelligent transportation systems, and drone control. Recently, the age of information (AoI) was proposed as a destination-centric metric to measure the information freshness in status update systems [1]-[3]. A status update packet contains the measured value of a monitored process and a time stamp representing the time when the sample was generated. Due to wireless channel access, channel errors, and fading etc., communicating a status update packet through the network experiences a random delay. If at a time instant $t$, the most recent status update available at the sink contains the time stamp $U(t)$, AoI is defined as the random process $\Delta(t)=t-U(t)$. Thus, the AoI measures for each sensor the time elapsed since the last available status update packet was generated at the sensor.

The work in [4] introduced a powerful technique, called stochastic hybrid systems (SHS), to calculate the average AoI.
In [5], the authors extended the SHS analysis to calculate the moment generating function (MGF) of the AoI. The SHS technique has been used to analyze the AoI in various queueing models [6]-[13].

In this paper, we consider a multi-source system with computation-intensive status updates in which the embedded information in each packet is not available until being processed by a server at the sink. An autonomous driving system in which images are status updates could be an example of the considered system model. In this system, further processing is needed at the sink to expose the embedded status information in the images. We introduce two packet management policies, namely, i) a preemptive policy and ii) a blocking policy, and derive the MGF of the AoI of each source under the policies. According to the preemptive policy, a new arriving packet preempts any possible packet that is currently under service regardless of the packet's source index. According to the blocking policy, when a server is busy at the arrival instant of a packet the arriving packet is blocked and cleared. We assume that the same preemptive/blocking policy is employed in both transmitter and sink servers. Numerical results are provided to assess the results.

The most related work to this paper is [14] where the authors derived the average AoI for a single-source system where the computation-intensive status updates are generated according to the zero-wait policy. According to the zero-wait policy, a new packet is generated immediately after the previous one is available at the sink. Whereas [14] derives the average AoI for a single-source setup, we consider multiple sources and derive the MGF of the AoI. In addition, [14] considers the zero-wait policy whereas, we consider random arrivals.

\section{SySTEM MODEL}

We consider a status update system consisting of two independent sources 1 , one transmitter server, one sink server, and one sink, as depicted in Fig. 1. Each source observes a random process at random time instants. The sink is

\footnotetext{
${ }^{1}$ Since we have multiple sources of Poisson arrivals, to evaluate the AoI of one source, we can consider two sources without loss of generality.
} 


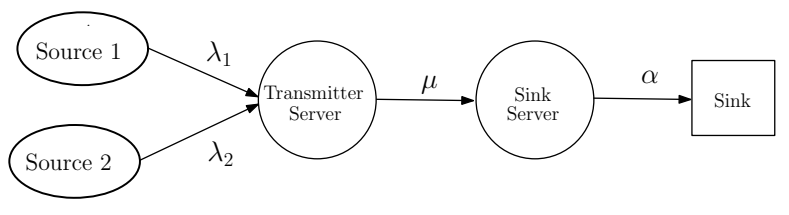

Fig. 1: The considered status update system.

interested in timely information about the status of these random processes. Status updates are transmitted as packets, containing the measured value of the monitored process and a time stamp representing the time when the sample was generated. We assume that the packets of sources 1 and 2 are generated according to the Poisson process with rates $\lambda_{1}$ and $\lambda_{2}$, respectively, and the packets are served by the transmitter server according to an exponentially distributed service time with mean $1 / \mu$. We assume that the embedded information in each packet is revealed to the sink only after being processed by the sink server which serves the packets according to an exponentially distributed service time with mean $1 / \alpha$.

Let $\rho_{1}=\lambda_{1} / \mu$ and $\rho_{2}=\lambda_{2} / \mu$ be the load of source 1 and 2 , respectively. The packet generation in the system follows the Poisson process with rate $\lambda=\lambda_{1}+\lambda_{2}$, and the overall load at the transmitter server is $\rho=\rho_{1}+\rho_{2}=\lambda / \mu$.

\section{A. Packet Management Policies}

According to the preemptive policy, a new arriving packet preempts any possible packet that is currently under service regardless of the packet's source index. According to the blocking policy, when a server is busy at the arrival instant of a packet the arriving packet is blocked and cleared. We assume that the same preemptive/blocking policy is employed in both transmitter and sink servers.

\section{B. Summary of the Main Results}

In this paper, we derive the MGF of the AoI for each source under both considered policies which are summarized by the following two theorems.

Theorem 1. The MGF of the AoI of source 1 under the preemptive policy is given as

$$
M_{\Delta_{1}}(s)=\frac{\rho_{1} \bar{\alpha}(\bar{s}-\bar{\alpha}-\rho-1)}{\bar{s}(1-\bar{s})(\rho-\bar{s})(\rho+1-\bar{s})+\bar{\alpha}^{2} \psi_{1}+\bar{\alpha} \psi_{2}},
$$

where $\bar{s}=s / \mu, \bar{\alpha}=\alpha / \mu$, and

$$
\begin{aligned}
\psi_{1}= & \rho \bar{s}-\rho_{1}+\bar{s}(1-\bar{s}), \\
\psi_{2}= & \rho_{1}^{2}(\bar{s}-1)+\rho_{1} \rho_{2}(2 \bar{s}-1)+\rho_{1}\left(-1+4 \bar{s}-3 \bar{s}^{2}\right)+ \\
& \rho_{2} \bar{s}\left(\rho_{2}+2-3 \bar{s}\right)+\bar{s}\left(1-3 \bar{s}+2 \bar{s}^{2}\right) .
\end{aligned}
$$

Proof. The proof of Theorem 1 appears in Section IV-A

Theorem 2. The MGF of the AoI of source 1 under the blocking policy is given by (3) (shown at the top of the next page), where $\bar{s}=s / \mu, \bar{\alpha}=\alpha / \mu$, and $\psi_{1}$ and $\psi_{2}$ are given in (2).

Proof. The proof of Theorem 2 appears in Section IV-B

\section{The SHS Technique to CAlCUlate MGF}

In the following, we briefly present how to use the SHS technique for our MGF analysis in Section IV] We refer the readers to [4], [5] for more details.

The SHS technique models a queueing system through the states $(q(t), \mathbf{x}(t))$, where $q(t) \in \mathcal{Q}=\{0,1, \ldots, m\}$ is a continuous-time finite-state Markov chain that describes the occupancy of the system and $\mathbf{x}(t)=\left[x_{0}(t) \cdots x_{n}(t)\right] \in \mathbb{R}^{1 \times(n+1)}$ is a continuous process that describes the evolution of age-related processes in the system. Following the approach in [4], [10], we label the source of interest as source 1 and employ the continuous process $\mathbf{x}(t)$ to track the age of source 1 updates at the sink.

The Markov chain $q(t)$ can be presented as a graph $(\mathcal{Q}, \mathcal{L})$ where each discrete state $q(t) \in \mathcal{Q}$ is a node of the chain and a (directed) link $l \in \mathcal{L}$ from node $q_{l}$ to node $q_{l}^{\prime}$ indicates a transition from state $q_{l} \in \mathcal{Q}$ to state $q_{l}^{\prime} \in \mathcal{Q}$.

A transition occurs when a packet arrives or departs in the system. Since the packets are generated according to the Poisson process and the service time is exponentially distributed, transition $l \in \mathcal{L}$ from state $q_{l}$ to state $q_{l}^{\prime}$ occurs with the exponential rate $\lambda^{(l)} \delta_{q_{l}, q(t)}$, where the Kronecker delta function $\delta_{q_{l}, q(t)}$ ensures that the transition $l$ occurs only when the discrete state $q(t)$ is equal to $q_{l}$. When a transition $l$ occurs, the discrete state $q_{l}$ changes to state $q_{l}^{\prime}$, and the continuous state $\mathbf{x}$ is reset to $\mathbf{x}^{\prime}$ according to a binary reset map matrix $\mathbf{A}_{l} \in \mathbb{B}^{(n+1) \times(n+1)}$ as $\mathbf{x}^{\prime}=\mathbf{x} \mathbf{A}_{l}$. In addition, as long as state $q(t)$ is unchanged we have $\dot{\mathbf{x}}(t) \triangleq \frac{\partial \mathbf{x}(t)}{\partial t}=\mathbf{1}$, where $\mathbf{1}$ is the row vector $[1 \cdots 1] \in \mathbb{R}^{1 \times(n+1)}$.

Note that unlike in a typical continuous-time Markov chain, a transition from a state to itself (i.e., a self-transition) is possible in $q(t) \in \mathcal{Q}$. In the case of a self-transition, a reset of the continuous state $\mathbf{x}$ takes place, but the discrete state remains the same. In addition, for a given pair of states $\bar{q}, \hat{q} \in \mathcal{Q}$, there may be multiple transitions $l$ and $l^{\prime}$ so that the discrete state changes from $\bar{q}$ to $\hat{q}$ but the transition reset maps $\mathbf{A}_{l}$ and $\mathbf{A}_{l^{\prime}}$ are different (for more details, see [4, Section III]).

To calculate the MGF of the AoI using the SHS technique, the state probabilities of the Markov chain, the correlation vector between the discrete state $q(t)$ and the continuous state $\mathbf{x}(t)$, and the correlation vector between the discrete state $q(t)$ and the exponential function $e^{s \mathbf{x}(t)}, s \in \mathbb{R}$, need to be defined. Let $\pi_{q}(t)$ denote the probability of being in state $q$ of the Markov chain. Let $\mathbf{v}_{q}(t)=\left[v_{q 0}(t) \cdots v_{q n}(t)\right] \in \mathbb{R}^{1 \times(n+1)}$ denote the correlation vector between the discrete state $q(t)$ and the continuous state $\mathbf{x}(t)$. Let $\mathbf{v}_{q}^{s}(t)=\left[v_{q 0}^{s}(t) \cdots v_{q n}^{s}(t)\right] \in$ $\mathbb{R}^{1 \times(n+1)}$ denote the correlation vector between the state $q(t)$ and the exponential function $e^{s \mathbf{x}(t)}$. Accordingly, we have

$$
\begin{gathered}
\pi_{q}(t)=\operatorname{Pr}(q(t)=q)=\mathbb{E}\left[\delta_{q, q(t)}\right], \quad \forall q \in \mathcal{Q}, \\
\mathbf{v}_{q}(t)=\left[v_{q 0}(t) \cdots v_{q n}(t)\right]=\mathbb{E}\left[\mathbf{x}(t) \delta_{q, q(t)}\right], \quad \forall q \in \mathcal{Q}, \\
\mathbf{v}_{q}^{s}(t)=\left[v_{q 0}^{s}(t) \cdots v_{q n}^{s}(t)\right]=\mathbb{E}\left[e^{s \mathbf{x}(t)} \delta_{q, q(t)}\right], \quad \forall q \in \mathcal{Q} .
\end{gathered}
$$




$$
M_{\Delta_{1}}(s)=\frac{\rho_{1} \bar{\alpha}^{2}(\bar{s}-\bar{\alpha}-\rho)(\rho+1-\bar{s})(\bar{\alpha}+\rho+1-\bar{s})}{(\bar{\alpha}-\bar{s})(1-\bar{s})(\bar{\alpha}+\rho)(\rho+1)\left(\bar{s}(1-\bar{s})(\rho-\bar{s})(\rho+1-\bar{s})+\bar{\alpha}^{2} \psi_{1}+\bar{\alpha} \psi_{2}\right)}
$$

Let $\mathcal{L}_{q}^{\prime}$ denote the set of incoming transitions and $\mathcal{L}_{q}$ denote the set of outgoing transitions for state $q$, defined as

$$
\mathcal{L}_{q}^{\prime}=\left\{l \in \mathcal{L}: q_{l}^{\prime}=q\right\}, \quad \mathcal{L}_{q}=\left\{l \in \mathcal{L}: q_{l}=q\right\}, \forall q \in \mathcal{Q} .
$$

Following the ergodicity assumption of the Markov chain $q(t)$ in the AoI analysis [4], [5], the state probability vector $\boldsymbol{\pi}(t)=$ $\left[\pi_{0}(t) \cdots \pi_{m}(t)\right]$ converges uniquely to the stationary vector $\overline{\boldsymbol{\pi}}=\left[\bar{\pi}_{0} \cdots \bar{\pi}_{m}\right]$ satisfying

$$
\begin{gathered}
\bar{\pi}_{q} \sum_{l \in \mathcal{L}_{q}} \lambda^{(l)}=\sum_{l \in \mathcal{L}_{q}^{\prime}} \lambda^{(l)} \bar{\pi}_{q_{l}}, \forall q \in \mathcal{Q}, \\
\sum_{q \in \mathcal{Q}} \bar{\pi}_{q}=1 .
\end{gathered}
$$

Further, it has been shown in [5, Theorem 1] that under the ergodicity assumption of the Markov chain $q(t)$, if we can find a non-negative limit $\overline{\mathbf{v}}_{q}=\left[\bar{v}_{q 0} \cdots \bar{v}_{q n}\right], \forall q \in \mathcal{Q}$, for the correlation vector $\mathbf{v}_{q}(t)$ satisfying

$$
\overline{\mathbf{v}}_{q} \sum_{l \in \mathcal{L}_{q}} \lambda^{(l)}=\bar{\pi}_{q} \mathbf{1}+\sum_{l \in \mathcal{L}_{q}^{\prime}} \lambda^{(l)} \overline{\mathbf{v}}_{q_{l}} \mathbf{A}_{l}, \quad \forall q \in \mathcal{Q},
$$

there exists $s_{0}>0$ such that for all $s<s_{0}, \mathbf{v}_{q}^{s}(t), \forall q \in \mathcal{Q}$, converges to $\overline{\mathbf{v}}_{q}^{s}$ that satisfies

$\overline{\mathbf{v}}_{q}^{s} \sum_{l \in \mathcal{L}_{q}} \lambda^{(l)}=s \overline{\mathbf{v}}_{q}^{s}+\sum_{l \in \mathcal{L}_{q}^{\prime}} \lambda^{(l)}\left[\overline{\mathbf{v}}_{q_{l}}^{s} \mathbf{A}_{l}+\bar{\pi}_{q_{l}} \mathbf{1} \hat{\mathbf{A}}_{l}\right], \quad \forall q \in \mathcal{Q}$,

where $\hat{\mathbf{A}}_{l} \in \mathbb{B}^{(n+1) \times(n+1)}$ is a binary matrix whose $k, j$ th element, $\hat{\mathbf{A}}_{l}(k, j)$, is given as

$\hat{\mathbf{A}}_{l}(k, j)= \begin{cases}1, & k=j, \text { and } j \text { th column of } \mathbf{A}_{l} \text { is a zero vector, } \\ 0, & \text { otherwise. }\end{cases}$

Finally, the MGF of the state $\mathbf{x}(t)$, which is calculated by $\mathbb{E}\left[e^{s \mathbf{x}(t)}\right]$, converges to the stationary vector [5, Theorem 1]

$$
\mathbb{E}\left[e^{s \mathbf{x}}\right]=\sum_{q \in \mathcal{Q}} \overline{\mathbf{v}}_{q}^{s}
$$

As (12) implies, if we set the first element of continuous state $\mathbf{x}(t)$ to represent the AoI of source 1 at the sink, the MGF of the AoI of source 1 at the sink converges to

$$
M_{\Delta_{1}}(s)=\sum_{q \in \mathcal{Q}} \bar{v}_{q 0}^{s} .
$$

Thus, the main challenge in calculating the MGF of the AoI of source 1 using the SHS technique reduces to deriving the first elements of correlation vectors $\overline{\mathbf{v}}_{q}^{s}, \forall q \in \mathcal{Q}$.

\section{AoI Analysis Using the SHS TECHNIQUE}

In this section, we use the SHS technique to calculate the MGF in 13 of each source under the considered packet management policies described in Section II-A

\section{A. Preemptive Policy}

The discrete states are $0=00,1=10,2=20,3=$ $01,4=11,5=21,6=02,7=12,8=22$. State $q=a_{1} a_{2}$ indicates that a packet of source $a_{1}$ is in the transmitter server and a packet of source $a_{2}$ is in the sink server. Note that $a_{1}=0$ (resp. $a_{2}=0$ ) indicates that the transmitter server (resp. the sink server) is idle.

The continuous process is $\mathbf{x}(t)=\left[x_{0}(t) x_{1}(t) x_{2}(t)\right]$, where $x_{0}(t)$ is the current AoI of source 1 at time instant $t$, $\Delta_{1}(t), x_{1}(t)$ encodes what $\Delta_{1}(t)$ would become if the packet that is in the sink server is delivered to the sink at time instant $t$, and $x_{2}(t)$ encodes what $\Delta_{1}(t)$ would become if the packet that is in the transmitter server is delivered to the sink at time instant $t$.

Recall that to calculate the MGF of the AoI of source 1 in (13) we need to find $\bar{v}_{q 0}^{s}, \forall q \in \mathcal{Q}$, which are the solution of the system of linear equations in 10 with variables $\overline{\mathbf{v}}_{q}^{s}, \forall q \in \mathcal{Q}$. To form the system of linear equations (9) and (10), for each state $q \in \mathcal{Q}$, we need to determine $\bar{\pi}_{q}$ and $\mathbf{A}_{l}$ and $\widehat{\mathbf{A}}_{l}$ for each incoming transition $l \in \mathcal{L}_{q}^{\prime}$, carried out next.

1) Calculation of $\bar{\pi}_{q} \forall q \in \mathcal{Q}$ : The Markov chain for the discrete state $q(t)$ is shown in Fig. 2 Using (7), (8), and the transition rates among the different states illustrated in Fig. 2 it can be shown that the stationary probability vector $\overline{\boldsymbol{\pi}}$ satisfies the following equations

$$
\begin{aligned}
& \lambda \bar{\pi}_{0}=\alpha \bar{\pi}_{3}+\alpha \bar{\pi}_{6} \\
& (\lambda+\mu) \bar{\pi}_{1}=\lambda_{1} \bar{\pi}_{0}+\lambda_{1} \bar{\pi}_{1}+\lambda_{1} \bar{\pi}_{2}+\alpha \bar{\pi}_{4}+\alpha \bar{\pi}_{7}, \\
& \left(\lambda_{1}+\mu\right) \bar{\pi}_{2}=\lambda_{2} \bar{\pi}_{0}+\lambda_{2} \bar{\pi}_{1}+\lambda_{2} \bar{\pi}_{2}+\alpha \bar{\pi}_{5}+\alpha \bar{\pi}_{8} \\
& (\lambda+\alpha) \bar{\pi}_{3}=\mu \bar{\pi}_{1}+\mu \bar{\pi}_{4}+\mu \bar{\pi}_{7} \\
& (\lambda+\mu+\alpha) \bar{\pi}_{4}=\lambda_{1} \bar{\pi}_{3}+\lambda_{1} \bar{\pi}_{4}+\lambda_{1} \bar{\pi}_{5} \\
& \left(\lambda_{1}+\mu+\alpha\right) \bar{\pi}_{5}=\lambda_{2} \bar{\pi}_{3}+\lambda_{2} \bar{\pi}_{4}+\lambda_{2} \bar{\pi}_{5} \\
& (\lambda+\alpha) \bar{\pi}_{6}=\mu \bar{\pi}_{2}+\mu \bar{\pi}_{5}+\mu \bar{\pi}_{8} \\
& (\lambda+\mu+\alpha) \bar{\pi}_{7}=\lambda_{1} \bar{\pi}_{6}+\lambda_{1} \bar{\pi}_{7}+\lambda_{1} \bar{\pi}_{8} \\
& \left(\lambda_{1}+\mu+\alpha\right) \bar{\pi}_{8}=\lambda_{2} \bar{\pi}_{6}+\lambda_{2} \bar{\pi}_{7}+\lambda_{2} \bar{\pi}_{8} \\
& \sum_{q \in \mathcal{Q}} \bar{\pi}_{q}=1 .
\end{aligned}
$$

Solving (14), the stationary probabilities are given as

$$
\begin{array}{ll}
\bar{\pi}_{0}=\frac{\alpha \mu}{\bar{\Psi}}, \bar{\pi}_{1}=\frac{\alpha \lambda_{1}(\alpha+\mu+\lambda)}{(\mu+\alpha) \bar{\Psi}}, & \bar{\pi}_{2}=\frac{\alpha \lambda_{2}(\alpha+\mu+\lambda)}{(\mu+\alpha) \bar{\Psi}}, \\
\bar{\pi}_{3}=\frac{\lambda_{1} \mu}{\bar{\Psi}}, \bar{\pi}_{4}=\frac{\lambda_{1}^{2} \mu}{(\mu+\alpha) \bar{\Psi}}, & \bar{\pi}_{5}=\frac{\lambda_{1} \lambda_{2} \mu}{(\mu+\alpha) \bar{\Psi}}, \\
\bar{\pi}_{6}=\frac{\lambda_{2} \mu}{\bar{\Psi}}, \bar{\pi}_{7}=\frac{\lambda_{1} \lambda_{2} \mu}{(\mu+\alpha) \bar{\Psi}}, & \bar{\pi}_{8}=\frac{\lambda_{2}^{2} \mu}{(\mu+\alpha) \bar{\Psi}}
\end{array}
$$

where $\bar{\Psi}=(\lambda+\alpha)(\lambda+\mu)$.

2) Transition reset map matrices $\mathbf{A}_{l}$ and $\hat{\mathbf{A}}_{l}$ : The transitions and their effects on the continuous state $\mathbf{x}(t)$ are 


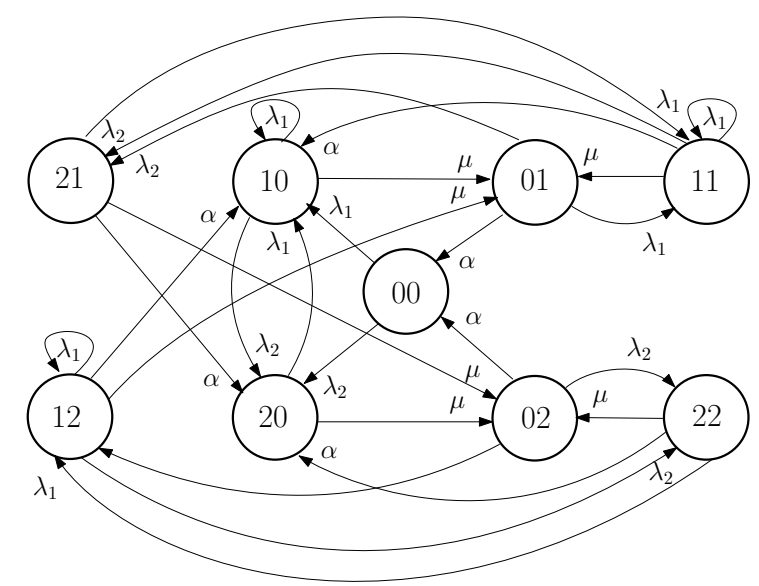

Fig. 2: The SHS Markov chain for the preemptive policy.

summarized in Table I. In the following, we explain the transitions in detail.

- $l=1$ : A source 1 packet arrives at the transmitter server. With this transition the AoI of source 1 does not change, i.e., $x_{0}^{\prime}=x_{0}$. This is because the arrival of source 1 packet does not yield an age reduction until it is delivered to the sink. Since the sink server is empty, $x_{1}$ becomes irrelevant to the AoI of source 1, and thus, it does not matter what is assigned to $x_{1}^{\prime}$. In this paper, when the system moves into a new state where $x_{i}$ is irrelevant, we set $x_{i}^{\prime}=x_{i}, i \in\{1,2\}$. Thus, for the transition $l=1$, we have $x_{1}^{\prime}=x_{1}$. Since the arriving source 1 packet is fresh and its age is zero, we have $x_{2}^{\prime}=0$. Finally, we have

$$
\mathbf{x}^{\prime}=\left[\begin{array}{lll}
x_{0} & x_{1} & x_{2}
\end{array}\right] \mathbf{A}_{1}=\left[\begin{array}{lll}
x_{0} & x_{1} & 0
\end{array}\right] .
$$

According to (16), it can be shown that the binary transition reset map matrix $\mathbf{A}_{1}$ is given by $\mathbf{A}_{1}=\left[\begin{array}{lll}1 & 0 & 0 \\ 0 & 1 & 0 \\ 0 & 0 & 0 \\ 0 & 0 & 0\end{array}\right]$. Then, by using (11), $\hat{\mathbf{A}}_{1}$ is given as $\hat{\mathbf{A}}_{1}=\left[\begin{array}{lll}0 & 0 & 0 \\ 0 & 0 & 0 \\ 0 & 0 & 1\end{array}\right]$. As it can be seen, when we have $\mathbf{x}^{\prime}$ for a transition $l \in \mathcal{L}$, it is easy to calculate $\mathbf{A}_{l}$ and $\hat{\mathbf{A}}_{l}$. Thus, for the rest of the transitions, we just explain the calculation of $\mathbf{x}^{\prime}$ and present the final expressions of $\mathbf{A}_{l}$ and $\hat{\mathbf{A}}_{l}$.

- $l=2$ : A source 2 packet arrives at the transmitter server. Similarly as for transition $l=1$, we have $x_{0}^{\prime}=x_{0}$ and $x_{1}^{\prime}=x_{1}$. Since the arriving packet is a source 2 packet, its delivery does not change the AoI of source 1, and thus, we have $x_{2}^{\prime}=x_{1}$.

- $l=3$ : A source 1 packet is in the transmitter server, the sink server is idle, and a source 1 packet arrives. According to the preemptive policy, the source 1 packet that is under service is preempted by the arriving packet. Similarly as for transition $l=1$, we have $x_{0}^{\prime}=x_{0}$ and $x_{1}^{\prime}=x_{1}$. Since the arriving source 1 packet is fresh and its age is zero, we have $x_{2}^{\prime}=0$. The reset maps
TABLE I: Table of transitions for the Markov chain in Fig. 2

\begin{tabular}{|c|c|c|c|c|c|c|c|c|c|}
\hline$l$ & $q_{l} \rightarrow q_{l}^{\prime}$ & $\lambda^{(l)}$ & $\mathbf{x} \mathbf{A}_{l}$ & \multicolumn{3}{|c|}{$\mathbf{A}_{l}$} & \multicolumn{3}{|c|}{$\hat{\mathbf{A}}_{l}$} \\
\hline 1 & $00 \rightarrow 10$ & $\lambda_{1}$ & {$\left[\begin{array}{lll}x_{0} & x_{1} & 0\end{array}\right]$} & & $\begin{array}{l}0 \\
1 \\
0\end{array}$ & $\left.\begin{array}{l}0 \\
0 \\
0\end{array}\right]$ & $\begin{array}{l}0 \\
0 \\
0\end{array}$ & $\begin{array}{l}0 \\
0 \\
0\end{array}$ & $\left.\begin{array}{l}0 \\
0 \\
1\end{array}\right]$ \\
\hline 2 & $00 \rightarrow 20$ & $\lambda_{2}$ & {$\left[\begin{array}{lll}x_{0} & x_{1} & x_{1}\end{array}\right]$} & 1 & $\begin{array}{l}0 \\
1 \\
0\end{array}$ & $\left.\begin{array}{l}0 \\
1 \\
0\end{array}\right]$ & $\begin{array}{l}0 \\
0 \\
0\end{array}$ & ( & $\left.\begin{array}{l}0 \\
0 \\
0\end{array}\right]$ \\
\hline 3 & $10 \rightarrow 10$ & $\lambda_{1}$ & {$\left[\begin{array}{lll}x_{0} & x_{1} & 0\end{array}\right]$} & 1 & $\begin{array}{l}0 \\
1 \\
0\end{array}$ & $\left.\begin{array}{l}0 \\
0 \\
0\end{array}\right]$ & 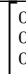 & s & $\left.\begin{array}{l}0 \\
0 \\
1\end{array}\right]$ \\
\hline 4 & $10 \rightarrow 20$ & $\lambda_{2}$ & {$\left[\begin{array}{lll}x_{0} & x_{1} & x_{1}\end{array}\right]$} & & $\begin{array}{l}0 \\
1 \\
0\end{array}$ & $\left.\begin{array}{l}0 \\
1 \\
0\end{array}\right]$ & & ( & $\left.\begin{array}{l}0 \\
0 \\
0\end{array}\right]$ \\
\hline 5 & $10 \rightarrow 01$ & $\mu$ & {$\left[\begin{array}{lll}x_{0} & x_{2} & x_{2}\end{array}\right]$} & 1 & $\begin{array}{l}0 \\
0 \\
1\end{array}$ & $\begin{array}{l}0 \\
0 \\
1\end{array}$ & $=0$ & ( & $\left.\begin{array}{l}0 \\
0 \\
0\end{array}\right]$ \\
\hline 6 & $20 \rightarrow 10$ & $\lambda_{1}$ & {$\left[\begin{array}{lll}x_{0} & x_{1} & 0\end{array}\right]$} & 1 & $\begin{array}{l}0 \\
1 \\
0\end{array}$ & $\left.\begin{array}{l}0 \\
0 \\
0\end{array}\right]$ & $\begin{array}{l}0 \\
0 \\
0\end{array}$ & c & $\begin{array}{l}0 \\
0 \\
1\end{array}$ \\
\hline 7 & $20 \rightarrow 02$ & $\mu$ & {$\left[\begin{array}{lll}x_{0} & x_{0} & x_{2}\end{array}\right]$} & 1 & $\begin{array}{l}1 \\
0 \\
0\end{array}$ & $\left.\begin{array}{l}0 \\
0 \\
1\end{array}\right]$ & $\begin{array}{l}0 \\
0 \\
0\end{array}$ & & $\left.\begin{array}{l}0 \\
0 \\
0\end{array}\right]$ \\
\hline 8 & $01 \rightarrow 11$ & $\lambda_{1}$ & {$\left[\begin{array}{lll}x_{0} & x_{1} & 0\end{array}\right]$} & 1 & $\begin{array}{l}0 \\
1 \\
0\end{array}$ & $\left.\begin{array}{l}0 \\
0 \\
0\end{array}\right]$ & & & $\left.\begin{array}{l}0 \\
0 \\
1\end{array}\right]$ \\
\hline 9 & $01 \rightarrow 21$ & $\lambda_{2}$ & {$\left[\begin{array}{lll}x_{0} & x_{1} & x_{1}\end{array}\right]$} & d & $\begin{array}{l}0 \\
1 \\
0\end{array}$ & $\begin{array}{l}0 \\
1 \\
0\end{array}$ & & & $\begin{array}{l}0 \\
0 \\
0\end{array}$ \\
\hline 10 & $01 \rightarrow 00$ & $\alpha$ & {$\left[\begin{array}{lll}x_{1} & x_{1} & x_{2}\end{array}\right]$} & 1 & $\begin{array}{l}1 \\
0 \\
0\end{array}$ & $\left.\begin{array}{l}0 \\
0 \\
1\end{array}\right]$ & {$\left[\begin{array}{l}0 \\
0 \\
0\end{array}\right.$} & 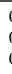 & $\left.\begin{array}{l}0 \\
0 \\
0\end{array}\right]$ \\
\hline 11 & $11 \rightarrow 11$ & $\lambda_{1}$ & {$\left[\begin{array}{lll}x_{0} & x_{1} & 0\end{array}\right]$} & 1 & $\begin{array}{l}0 \\
1 \\
0\end{array}$ & $\left.\begin{array}{l}0 \\
0 \\
0\end{array}\right]$ & 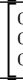 & 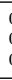 & $\left.\begin{array}{l}0 \\
0 \\
1\end{array}\right]$ \\
\hline 12 & $11 \rightarrow 21$ & $\lambda_{2}$ & {$\left[\begin{array}{lll}x_{0} & x_{1} & x_{1}\end{array}\right]$} & 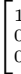 & $\begin{array}{l}0 \\
1 \\
0\end{array}$ & $\left.\begin{array}{l}0 \\
1 \\
0\end{array}\right]$ & & 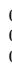 & $\left.\begin{array}{l}0 \\
0 \\
0\end{array}\right]$ \\
\hline 13 & $11 \rightarrow 01$ & $\mu$ & {$\left[\begin{array}{lll}x_{0} & x_{2} & x_{2}\end{array}\right]$} & 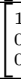 & $\begin{array}{l}0 \\
0 \\
1\end{array}$ & $\left.\begin{array}{l}0 \\
0 \\
1\end{array}\right]$ & 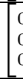 & & $\left.\begin{array}{l}0 \\
0 \\
0\end{array}\right]$ \\
\hline 14 & $11 \rightarrow 10$ & $\alpha$ & {$\left[\begin{array}{lll}x_{1} & x_{1} & x_{2}\end{array}\right]$} & 1 & $\begin{array}{l}0 \\
1 \\
0\end{array}$ & $\left.\begin{array}{l}0 \\
0 \\
1\end{array}\right]$ & 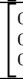 & & $\left.\begin{array}{l}0 \\
0 \\
0\end{array}\right]$ \\
\hline 15 & $21 \rightarrow 11$ & $\lambda_{1}$ & {$\left[\begin{array}{lll}x_{0} & x_{1} & 0\end{array}\right]$} & d & $\begin{array}{l}0 \\
1 \\
0\end{array}$ & $\left.\begin{array}{l}0 \\
0 \\
0\end{array}\right]$ & 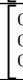 & & $\left.\begin{array}{l}0 \\
0 \\
1\end{array}\right]$ \\
\hline 16 & $21 \rightarrow 02$ & $\mu$ & {$\left[\begin{array}{lll}x_{0} & x_{0} & x_{2}\end{array}\right]$} & $\begin{array}{l}1 \\
0 \\
0\end{array}$ & $\begin{array}{l}1 \\
0 \\
0\end{array}$ & {$\left[\begin{array}{l}0 \\
0 \\
1\end{array}\right]$} & & 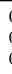 & $\left.\begin{array}{l}0 \\
0 \\
0\end{array}\right]$ \\
\hline 17 & $21 \rightarrow 20$ & $\alpha$ & {$\left[\begin{array}{lll}x_{1} & x_{1} & x_{1}\end{array}\right]$} & {$\left[\begin{array}{l}0 \\
1 \\
0\end{array}\right.$} & $\begin{array}{l}0 \\
1 \\
0\end{array}$ & $\left.\begin{array}{l}0 \\
1 \\
0\end{array}\right]$ & 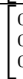 & 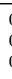 & $\left.\begin{array}{l}0 \\
0 \\
0\end{array}\right]$ \\
\hline 18 & $02 \rightarrow 12$ & $\lambda_{1}$ & {$\left[\begin{array}{lll}x_{0} & x_{0} & 0\end{array}\right]$} & 1 & $\begin{array}{l} \\
0 \\
0 \\
\end{array}$ & $\begin{array}{l}0 \\
0 \\
0\end{array}$ & 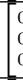 & 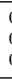 & $\begin{array}{l}0 \\
0 \\
1 \\
\end{array}$ \\
\hline 19 & $02 \rightarrow 22$ & $\lambda_{2}$ & {$\left[\begin{array}{lll}x_{0} & x_{0} & x_{0}\end{array}\right]$} & 1 & $\begin{array}{l}1 \\
0 \\
0 \\
0\end{array}$ & $\begin{array}{l}1 \\
0 \\
0\end{array}$ & 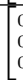 & 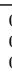 & $\begin{array}{l}0 \\
0 \\
0\end{array}$ \\
\hline 20 & $02 \rightarrow 00$ & $\alpha$ & {$\left[\begin{array}{lll}x_{0} & x_{1} & x_{2}\end{array}\right]$} & 1 & $\begin{array}{l}0 \\
1 \\
0\end{array}$ & $\begin{array}{l}0 \\
0 \\
1 \\
\end{array}$ & 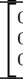 & 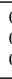 & $\left.\begin{array}{l}0 \\
0 \\
0\end{array}\right]$ \\
\hline 21 & $12 \rightarrow 12$ & $\lambda_{1}$ & {$\left[\begin{array}{lll}x_{0} & x_{0} & 0\end{array}\right]$} & 1 & $\begin{array}{l}1 \\
0 \\
0\end{array}$ & $\left.\begin{array}{l}0 \\
0 \\
0\end{array}\right]$ & 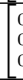 & & $\left.\begin{array}{l}0 \\
0 \\
1\end{array}\right]$ \\
\hline 22 & $12 \rightarrow 22$ & $\lambda_{2}$ & {$\left[\begin{array}{lll}x_{0} & x_{0} & x_{0}\end{array}\right]$} & 1 & $\begin{array}{l}1 \\
0 \\
0\end{array}$ & $\left.\begin{array}{l}1 \\
0 \\
0\end{array}\right]$ & 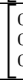 & & $\left.\begin{array}{l}0 \\
0 \\
0\end{array}\right]$ \\
\hline 23 & $12 \rightarrow 01$ & $\mu$ & {$\left[\begin{array}{lll}x_{0} & x_{2} & x_{2}\end{array}\right]$} & 1 & $\begin{array}{l}0 \\
0 \\
1\end{array}$ & $\left.\begin{array}{l}0 \\
0 \\
1\end{array}\right]$ & 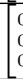 & 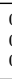 & $\left.\begin{array}{l}0 \\
0 \\
0\end{array}\right]$ \\
\hline 24 & $12 \rightarrow 10$ & $\alpha$ & {$\left[\begin{array}{lll}x_{0} & x_{1} & x_{2}\end{array}\right]$} & 1 & $\begin{array}{l}0 \\
1 \\
0\end{array}$ & $\begin{array}{l}0 \\
0 \\
1\end{array}$ & s & 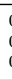 & $\left.\begin{array}{l}0 \\
0 \\
0\end{array}\right]$ \\
\hline 25 & $22 \rightarrow 12$ & $\lambda_{1}$ & {$\left[\begin{array}{lll}x_{0} & x_{0} & 0\end{array}\right]$} & 1 & $\begin{array}{l}1 \\
0 \\
0\end{array}$ & $\begin{array}{l}0 \\
0 \\
0\end{array}$ & , & & $\begin{array}{l}0 \\
0 \\
1\end{array}$ \\
\hline 26 & $22 \rightarrow 02$ & $\mu$ & {$\left[\begin{array}{lll}x_{0} & x_{0} & x_{2}\end{array}\right]$} & 1 & $\begin{array}{l}1 \\
0 \\
0\end{array}$ & . $\begin{array}{l}0 \\
0 \\
\end{array}$ & 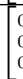 & & $\left.\begin{array}{l}0 \\
0 \\
0\end{array}\right]$ \\
\hline 27 & $22 \rightarrow 20$ & $\alpha$ & {$\left[\begin{array}{lll}x_{0} & x_{1} & x_{1}\end{array}\right]$} & $\begin{array}{l}1 \\
0 \\
0\end{array}$ & $\begin{array}{l}0 \\
1 \\
0\end{array}$ & 0 & & & $\left.\begin{array}{l}0 \\
0 \\
0\end{array}\right]$ \\
\hline
\end{tabular}


of transitions $l=11$ and $l=21$ can be derived similarly. - $l=4$ : A source 1 packet is in the transmitter server, the sink server is idle, and a source 2 packet arrives. According to the preemptive policy, the source 1 packet that is under service is preempted by the arriving packet. Similarly as for transition $l=1$, we have $x_{0}^{\prime}=x_{0}$ and $x_{1}^{\prime}=x_{1}$. Since the arriving packet is a source 2 packet, its delivery does not change the AoI of source 1 , and thus we have $x_{2}^{\prime}=x_{1}$. The reset maps of transitions $l=12$ and $l=22$ can be derived similarly.

- $l=5$ : The sink server is idle, and the source 1 packet in the transmitter server completes service and moves to the sink server. With this transition, we have $x_{0}^{\prime}=x_{0}$ because this transition does not change the AoI at the sink. Since the source 1 packet moves to the sink server and its delivery would reduce the AoI to $x_{2}$, we have $x_{1}^{\prime}=x_{2}$. Since the transmitter server is empty, $x_{2}$ becomes irrelevant, and thus, we have $x_{2}^{\prime}=x_{2}$. The reset maps of transitions $l=13$ and $l=23$ can be derived similarly.

- $l=6$ : A source 2 packet is in the transmitter server, the sink server is idle, and a source 1 packet arrives. According to the preemptive policy, the source 2 packet that is under service is preempted by the arriving packet. Similarly as for transition $l=1$, we have $x_{0}^{\prime}=x_{0}$ and $x_{1}^{\prime}=x_{1}$. Since the arriving source 1 packet is fresh and its age is zero, we have $x_{2}^{\prime}=0$. The reset maps of transitions $l=15$ and $l=25$ can be derived similarly.

- $l=7$ : The sink server is idle and the source 2 packet in the transmitter server completes service and moves to the sink server. With this transition, we have $x_{0}^{\prime}=x_{0}$, and since the source 2 packet delivery does not change the AoI of source 1 , we have $x_{1}^{\prime}=x_{0}$. Since the transmitter server is empty, $x_{2}$ becomes irrelevant, and thus, we have $x_{2}^{\prime}=x_{2}$. The reset maps of transitions $l=16$ and $l=26$ can be derived similarly.

- $l=8$ : The transmitter server is idle, a source 1 packet is in the sink server, and a source 1 packet arrives. With this transition, we have $x_{0}^{\prime}=x_{0}$. The delivery of the packet in the sink server would reduce the AoI to $x_{1}$ thus, we have $x_{1}^{\prime}=x_{1}$. Since the arriving source 1 packet is fresh and its age is zero, we have $x_{2}^{\prime}=0$. The reset maps of transition $l=18$ can be derived similarly.

- $l=9$ : The transmitter server is idle, a source 1 packet is in the sink server, and a source 2 packet arrives. Similarly as for transition $l=8$, we have $x_{0}^{\prime}=x_{0}$ and $x_{1}^{\prime}=x_{1}$. Since the arriving packet is a source 2 packet, its delivery does not change the AoI of source 1, and thus we have $x_{2}^{\prime}=x_{1}$. The reset maps of transition $l=19$ can be derived similarly.

- $l=10$ : The transmitter server is idle and the source 1 packet in the sink server completes service and is delivered to the sink. With this transition, we have $x_{0}^{\prime}=x_{1}$. Since the servers become idle we have $x_{1}^{\prime}=x_{1}$ and $x_{2}^{\prime}=x_{2}$. The reset maps of transitions $l \in$ $\{14,17,20,24,27\}$ can be derived similarly.
3) Calculation of the MGF of the AoI: Recall from Section III that to calculate MGF of the AoI, we need to first ensure whether we can find non-negative vectors $\overline{\mathbf{v}}_{q}=\left[\bar{v}_{q 0} \cdots \bar{v}_{q n}\right], \forall q \in \mathcal{Q}$, that satisfy [9]. Having derived the matrices $\mathbf{A}_{l}$ for all transitions (see Table I), we can form the system of linear equations in (9). It can be shown that the system of linear equations in (9) has a non-negative solution. Consequently, using the derived $\mathbf{A}_{l}$ and $\hat{\mathbf{A}}_{l}$ for the transitions in Table I, we can form the system of linear equations in (10). Finally, by solving the formed system of linear equations, the values of $\bar{v}_{q 0}^{s}, \forall q \in \mathcal{Q}$, are calculated as presented in Appendix A Substituting $\bar{v}_{q 0}^{s}, \forall q \in \mathcal{Q}$ into (13) results the MGF of the AoI of source 1 under the preemptive policy, given in Theorem 1 .

\section{B. Blocking Policy}

The elements of discrete state space $\mathcal{Q}$ are $0=\mathrm{II}, 1=$ $\mathrm{IB}, 2=\mathrm{BI}, 3=\mathrm{BB}$, where "I" stands for "idle" and "B" stands for "busy". State $0=$ II indicates that both the transmitter and sink servers are idle; $1=$ IB indicates that the transmitter server is idle and there is a packet (regardless of the source) under service in the sink server; $2=\mathrm{BI}$ indicates that there is a packet under service in the transmitter server and the sink server is idle; and $3=\mathrm{BB}$ indicates that both the transmitter and sink servers are busy. The continuous state space is the same as that for the preemptive policy.

Next, we determine $\bar{\pi}_{q}, \forall q \in \mathcal{Q}, \mathbf{A}_{l}$, and $\hat{\mathbf{A}}_{l}$ for each incoming transition $l \in \mathcal{L}_{q}^{\prime}$ which are needed to form the system of linear equations (9) and (10).

1) Calculation of $\bar{\pi}_{q} \forall q \in \mathcal{Q}$ : The Markov chain for the discrete state $q(t)$ is shown in Fig. 3. Using (7) and the transition rates among the different states illustrated in Fig. 3 it can be shown that the stationary probability vector $\overline{\boldsymbol{\pi}}$ satisfies $\overline{\boldsymbol{\pi}} \mathbf{D}=\overline{\boldsymbol{\pi}} \mathbf{Q}$ with $\mathbf{D}=\operatorname{diag}\left[\lambda, \lambda_{1}+\mu, \lambda+\alpha, \lambda_{1}+\mu+\alpha\right]$ and

$$
\mathbf{Q}=\left[\begin{array}{cccc}
0 & \lambda & 0 & 0 \\
0 & \lambda_{1} & \mu & 0 \\
\alpha & 0 & 0 & \lambda \\
0 & \alpha & \mu & \lambda_{1}
\end{array}\right]
$$

Using the above $\bar{\pi} \mathbf{D}=\bar{\pi} \mathbf{Q}$ and $\sum_{q \in \mathcal{Q}} \bar{\pi}_{q}=1$ in (8), the stationary probabilities are given as

$$
\overline{\boldsymbol{\pi}}=\frac{1}{(\lambda+\mu)(\lambda+\alpha)}\left[\alpha \mu, \frac{\alpha \lambda(\alpha+\lambda+\mu)}{\alpha+\mu}, \mu \lambda, \frac{\mu \lambda^{2}}{\alpha+\mu}\right] .
$$

2) Transition reset map matrices $\mathbf{A}_{l}$ and $\hat{\mathbf{A}}_{l}$ : The transitions, their effects on the continuous state $\mathbf{x}(t)$, and the values of $\mathbf{A}_{l}$ and $\hat{\mathbf{A}}_{l}$ are summarized in Table II Following the arguments of Section IV-A, we simply present the final values of $\mathbf{A}_{l}$ and $\hat{\mathbf{A}}_{l}$ in Table $[\mathrm{II}$

- $l=1$ : A source 1 packet arrives at the transmitter server. We have $x_{0}^{\prime}=x_{0}$, because there is no departure at the sink server. Since the sink server is empty, $x_{1}$ becomes irrelevant to the AoI of source 1, and thus, we have $x_{1}^{\prime}=$ $x_{1}$. Since the arriving source 1 packet is fresh and its age is zero, we have $x_{2}^{\prime}=0$. The reset maps of transition $l=6$ can be derived similarly. 


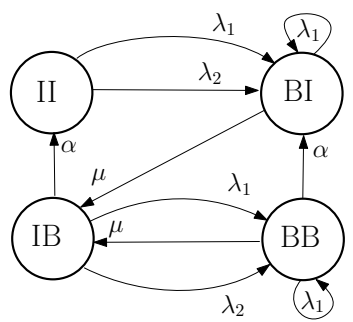

Fig. 3: The SHS Markov chain for the blocking policy.

TABLE II: Table of transitions for the Markov chain in Fig. 3

\begin{tabular}{|c|c|c|c|c|c|c|c|c|c|}
\hline$l$ & $q_{l} \rightarrow q_{l}^{\prime}$ & $\lambda^{(l)}$ & $\mathbf{x} \mathbf{A}_{l}$ & \multicolumn{3}{|c|}{$\mathbf{A}_{l}$} & \multicolumn{3}{|c|}{$\hat{\mathbf{A}}_{l}$} \\
\hline 1 & $\mathrm{II} \rightarrow \mathrm{BI}$ & $\lambda_{1}$ & {$\left[\begin{array}{lll}x_{0} & x_{1} & 0\end{array}\right]$} & & 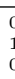 & $\begin{array}{l}0 \\
0 \\
0\end{array}$ & & & $\begin{array}{l}0 \\
0 \\
1\end{array}$ \\
\hline 2 & $\mathrm{II} \rightarrow \mathrm{BI}$ & $\lambda_{2}$ & {$\left[\begin{array}{lll}x_{0} & x_{1} & x_{1}\end{array}\right]$} & & . & $\begin{array}{l}0 \\
1 \\
0\end{array}$ & & & $\begin{array}{l}0 \\
0 \\
0\end{array}$ \\
\hline 3 & $\mathrm{BI} \rightarrow \mathrm{BI}$ & $\lambda_{1}$ & {$\left[\begin{array}{lll}x_{0} & x_{1} & x_{2}\end{array}\right]$} & & ( & $\begin{array}{l}0 \\
0 \\
1\end{array}$ & & & 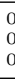 \\
\hline 4 & $\mathrm{BI} \rightarrow \mathrm{IB}$ & $\mu$ & {$\left[\begin{array}{lll}x_{0} & x_{2} & x_{2}\end{array}\right]$} & & 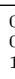 & $\begin{array}{l}0 \\
0 \\
1\end{array}$ & & & $\begin{array}{l}0 \\
0 \\
0\end{array}$ \\
\hline 5 & $\mathrm{IB} \rightarrow \mathrm{II}$ & $\alpha$ & {$\left[\begin{array}{lll}x_{1} & x_{1} & x_{2}\end{array}\right]$} & & . & $\begin{array}{l}0 \\
0 \\
1\end{array}$ & & & $\begin{array}{l}0 \\
0 \\
0\end{array}$ \\
\hline 6 & $\mathrm{IB} \rightarrow \mathrm{BB}$ & $\lambda_{1}$ & {$\left[\begin{array}{lll}x_{0} & x_{1} & 0\end{array}\right]$} & & 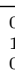 & $\begin{array}{l}0 \\
0 \\
0\end{array}$ & & & $\begin{array}{l}0 \\
0 \\
1\end{array}$ \\
\hline 7 & $\mathrm{IB} \rightarrow \mathrm{BB}$ & $\lambda_{2}$ & {$\left[\begin{array}{lll}x_{0} & x_{1} & x_{1}\end{array}\right]$} & & c & $\begin{array}{l}0 \\
1 \\
0\end{array}$ & & & $\begin{array}{l}0 \\
0 \\
0\end{array}$ \\
\hline 8 & $\mathrm{BB} \rightarrow \mathrm{BB}$ & $\lambda_{1}$ & {$\left[\begin{array}{lll}x_{0} & x_{1} & x_{2}\end{array}\right]$} & & c & $\begin{array}{l}0 \\
0 \\
1\end{array}$ & & & $\begin{array}{c}0 \\
0 \\
0 \\
0\end{array}$ \\
\hline 9 & $\mathrm{BB} \rightarrow \mathrm{IB}$ & $\mu$ & {$\left[\begin{array}{lll}x_{0} & x_{1} & x_{2}\end{array}\right]$} & & 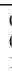 & $\begin{array}{l}0 \\
0 \\
0\end{array}$ & & & $\begin{array}{l}0 \\
0 \\
0\end{array}$ \\
\hline 10 & $\mathrm{BB} \rightarrow \mathrm{BI}$ & $\alpha$ & {$\left[\begin{array}{lll}x_{1} & x_{1} & x_{2}\end{array}\right]$} & & 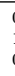 & $\begin{array}{l}0 \\
0 \\
1\end{array}$ & & & $\overline{0}$ \\
\hline
\end{tabular}

- $l=2$ : A source 2 packet arrives at the transmitter server. Similarly as for transition $l=1$, we have $x_{0}^{\prime}=x_{0}$ and $x_{1}^{\prime}=x_{1}$. Since the arriving packet is a source 2 packet, its delivery does not change the AoI of source 1 , and thus we have $x_{2}^{\prime}=x_{1}$. The reset maps of transition $l=7$ can be derived similarly.

- $l=3$ : A packet is in the transmitter server, the sink server is idle, and a source 1 packet arrives. According to the blocking policy the arriving packet is blocked and cleared. Similarly as for transition $l=1$, we have $x_{0}^{\prime}=x_{0}$ and $x_{1}^{\prime}=x_{1}$. The delivery of the packet in the transmitter server to the sink would reduce the AoI to $x_{2}$ and thus, $x_{2}^{\prime}=x_{2}$. The reset maps of transition $l=8$ can be derived similarly.

- $l=4$ : A packet in the transmitter server completes service and moves to the sink server. In this transition, we have $x_{0}^{\prime}=x_{0}$ because this transition does not change the AoI at the sink. Since the packet moves to the sink server, we have $x_{1}^{\prime}=x_{2}$. Since the transmitter server is empty, $x_{2}$ becomes irrelevant, and thus, we have $x_{2}^{\prime}=x_{2}$. The reset maps of transition $l=9$ can be derived similarly.

- $l=5$ : A packet in the sink server completes service and is delivered to the sink. With this transition, we have $x_{0}^{\prime}=$ $x_{1}$. Since with this transition the both servers become idle we have $x_{1}^{\prime}=x_{1}$ and $x_{2}^{\prime}=x_{2}$. The reset maps of transition $l=10$ can be derived similarly.

3) Calculation of the MGF of the AoI: Having derived the matrices $\mathbf{A}_{l}$ for all transitions (see Table II), we can form the system of linear equations in (9). It can be shown that the system of linear equations in $(9)$ has a non-negative solution. Using the derived $\mathbf{A}_{l}$ and $\hat{\mathbf{A}}_{l}$ for the transitions in Table $\mathrm{II}$ we can form the system of linear equations in 10 . By solving the system of linear equations, values of $\bar{v}_{q 0}^{s}, \forall q \in \mathcal{Q}$, are given as

$$
\begin{aligned}
\bar{v}_{00}^{s} & =\frac{-\bar{\alpha}^{2} \rho_{1}(\bar{\alpha}+\rho+1-\bar{s})}{(\bar{\alpha}+\rho)(\rho+1) \Psi}, \\
\bar{v}_{10}^{s} & =\frac{-\bar{\alpha}^{2} \rho_{1} \rho(\bar{\alpha}+\rho+1-\bar{s})}{(\bar{\alpha}+1-\bar{s})(1-\bar{s})(\bar{\alpha}+\rho)(\rho+1) \Psi}, \\
\bar{v}_{20}^{s} & =\frac{-\bar{\alpha}^{2} \rho_{1} \rho(\bar{\alpha}+\rho+1-\bar{s})}{(\bar{\alpha}-\bar{s})(1-\bar{s})(\bar{\alpha}+\rho)(\rho+1) \Psi}, \\
\bar{v}_{30}^{s} & =\frac{-\bar{\alpha}^{2} \rho_{1} \rho^{2}(\bar{\alpha}+\rho+1-\bar{s})}{(\bar{\alpha}-\bar{s})(\bar{\alpha}+1-\bar{s})(1-\bar{s})(\bar{\alpha}+\rho)(\rho+1) \Psi},
\end{aligned}
$$

where $\bar{s}=s / \mu, \bar{\alpha}=\alpha / \mu$, and $\Psi=\bar{s}(1-\bar{s})(\rho-\bar{s})(\rho+1-$ $\bar{s})+\bar{\alpha}^{2} \psi_{1}+\bar{\alpha} \psi_{2}$, where $\psi_{1}$ and $\psi_{2}$ are given in (2).

Substituting $\bar{v}_{q 0}^{s}, \forall q \in \mathcal{Q}$, in (18) into (13) results in the MGF of the AoI of source 1 under the blocking policy, given in Theorem 3

\section{VALIDATIONS AND NUMERICAL RESUlTS}

By using the derived MGFs the $m$ th moment of the AoI of source $1, \Delta_{1}^{(m)}$, is given by $\Delta_{1}^{(m)}=\left.\frac{\mathrm{d}^{m}\left(M_{\Delta_{1}}(s)\right)}{\mathrm{d} s^{m}}\right|_{s=0}$. It can be shown that the average AoI under the preemptive policy derived by using the MGF generalizes the existing results in [7] and [4]. Specifically, when we set $\lambda_{2}=0$, the average AoI follows the result in [7]; and as $\alpha \rightarrow \infty$ or $\mu \rightarrow \infty$, the average AoI coincides with the average AoI expression under the LCFS-S policy introduced in [4].

Fig. 4 illustrates the average AoI of source 1 and its standard deviation $(\sigma)$ as a function of $\lambda_{1}$ under the considered policies for $\mu=1, \alpha=1$, and $\lambda=5$. Simulated curves are shown as a verification of the derived theoretical results. From the figure, it can be seen that the preemptive policy outperforms the blocking policy. We would like to point out that based on our experiments (not included here due to space limitation), this conclusion appears to hold true for any choice of the parameters. In addition, it can be seen that the first moment $\left(\Delta_{1}\right)$ and the standard deviation $(\sigma)$ of the AoI increase by the same amount as $\rho_{1}$ decreases; thus their difference remains approximately constant.

\section{Conclusions}

We considered a multi-source system with computationintensive status updates and derived the MGF of the AoI under two packet management policies using the SHS technique. Using the derived MGFs we evaluated the average AoI and its standard deviation. The results showed that the preemptive policy provides better performance than that of the blocking policy. 


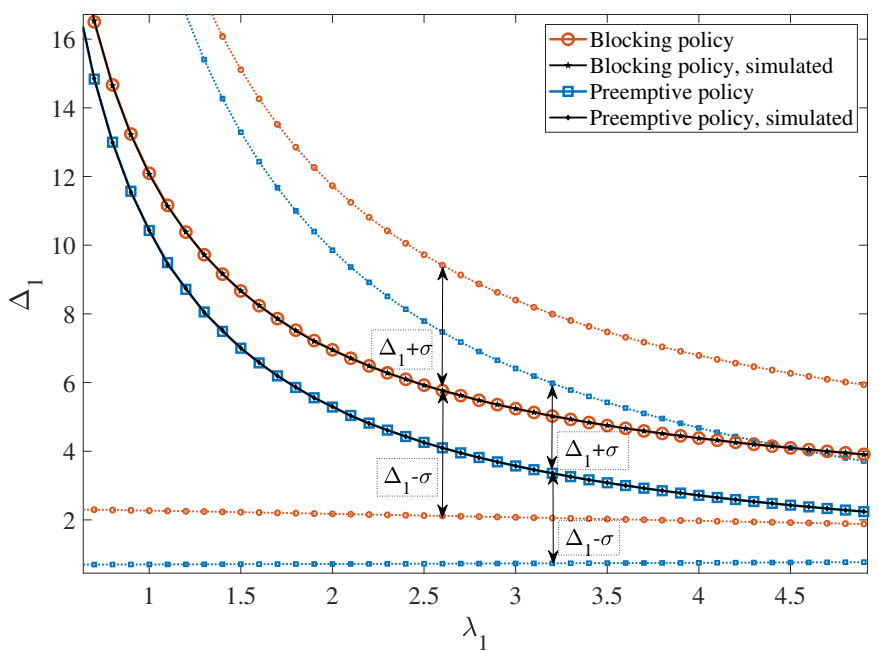

Fig. 4: The average AoI of source 1 and its standard deviation $(\sigma)$ as a function of $\rho_{1}$ under the two packet management policies for $\mu=1, \alpha=1$, and $\lambda=5$. The dashed lines visualize the standard deviation of the AoI as $\Delta_{1}+\sigma$ and $\Delta_{1}-\sigma$.

\section{APPENDIX A VAlues of $\bar{v}_{q 0}^{s}$ FOR THe Preemptive Policy}

$$
\begin{aligned}
& \bar{v}_{00}^{s}= \\
& \frac{\rho_{1} \bar{\alpha}(\bar{s}-\bar{\alpha})(1-\bar{s})(\bar{\alpha}+\rho+1-\bar{s})}{(\bar{\alpha}+\rho-\bar{s})(1+\rho-\bar{s})\left(\bar{s}(1-\bar{s})(\rho-\bar{s})(\rho+1-\bar{s})+\bar{\alpha}^{2} \psi_{1}+\bar{\alpha} \psi_{2}\right)}, \\
& \bar{v}_{10}^{s}=\frac{\bar{v}_{00}^{s} \rho_{1} \hat{\Psi}}{(\bar{\alpha}+\rho-\bar{s})(1-\bar{s})(\bar{\alpha}+\rho+1-\bar{s})}, \\
& \bar{v}_{20}^{s}=\frac{\bar{v}_{00}^{s} \rho_{2} \hat{\Psi}}{(\bar{\alpha}+\rho-\bar{s})(1-\bar{s})(\bar{\alpha}+\rho+1-\bar{s})}, \\
& \bar{v}_{30}^{s}=\frac{\bar{v}_{00}^{s} \rho_{1}(\bar{\alpha}+\rho+1-2 \bar{s})}{(\bar{\alpha}+1-\bar{s})(1-\bar{s})(\bar{\alpha}+\rho+1-\bar{s})}, \\
& \bar{v}_{40}^{s}=\frac{\bar{v}_{00}^{s} \rho_{1}^{2}(\bar{\alpha}+\rho+1-2 \bar{s})}{(\bar{\alpha}+1-\bar{s})(\bar{\alpha}-\bar{s})(1-\bar{s})(\bar{\alpha}+\rho+1-\bar{s})}, \\
& \bar{v}_{50}^{s}=\frac{\bar{v}_{00}^{s} \rho_{1} \rho_{2}(\bar{\alpha}+\rho+1-2 \bar{s})}{(\bar{\alpha}+1-\bar{s})(\bar{\alpha}-\bar{s})(1-\bar{s})(\bar{\alpha}+\rho+1-\bar{s})}, \\
& \bar{v}_{60}^{s}=\frac{\bar{v}_{00}^{s} \rho_{2}(\bar{\alpha}+\rho+1-2 \bar{s})}{(\bar{\alpha}-\bar{s})(1-\bar{s})(\bar{\alpha}+\rho+1-\bar{s})}, \\
& \bar{v}_{70}^{s}=\frac{\bar{v}_{00}^{s} \rho_{1} \rho_{2}(\bar{\alpha}+\rho+1-2 \bar{s})}{(\bar{\alpha}+1-\bar{s})(\bar{\alpha}-\bar{s})(1-\bar{s})(\bar{\alpha}+\rho+1-\bar{s})}, \\
& \bar{v}_{80}^{s}=\frac{\bar{v}_{00}^{s} \rho_{2}^{2}(\bar{\alpha}+\rho+1-2 \bar{s})}{(\bar{\alpha}+1-\bar{s})(\bar{\alpha}-\bar{s})(1-\bar{s})(\bar{\alpha}+\rho+1-\bar{s})},
\end{aligned}
$$

where $\hat{\Psi}=\bar{\alpha}^{2}+\bar{\alpha}(2 \rho-2-3 \bar{s})+\rho^{2}+\rho_{1}\left(2 \rho_{2}+2-3 \bar{s}\right)+$ $\rho_{2}^{2}+\rho_{2}(2-3 \bar{s})+1-3 \bar{s}+2 \bar{s}^{2}$, and $\psi_{1}$ and $\psi_{2}$ are given in (2).

\section{REFERENCES}

[1] S. Kaul, M. Gruteser, V. Rai, and J. Kenney, "Minimizing age of information in vehicular networks," in Proc. Commun. Society. Conf. on Sensor, Mesh and Ad Hoc Commun. and Net., Salt Lake City, UT, USA, Jun. 27-30, 2011, pp. 350-358.
[2] S. Kaul, R. Yates, and M. Gruteser, "Real-time status: How often should one update?" in Proc. IEEE Int. Conf. on Computer Commun. (INFOCOM), Orlando, FL, USA, Mar. 25-30, 2012, pp. 2731-2735.

[3] S. K. Kaul, R. D. Yates, and M. Gruteser, "Status updates through queues," in Proc. Conf. Inform. Sciences Syst. (CISS), Princeton, NJ, USA, Mar. 21-23, 2012, pp. 1-6.

[4] R. D. Yates and S. K. Kaul, "The age of information: Real-time status updating by multiple sources," IEEE Trans. Inform. Theory, vol. 65, no. 3, pp. 1807-1827, Mar. 2019.

[5] R. D. Yates, "The age of information in networks: Moments, distributions, and sampling," IEEE Trans. Inform. Theory, vol. 66, no. 9, pp. 5712-5728, May 2020.

[6] S. K. Kaul and R. D. Yates, "Age of information: Updates with priority," in Proc. IEEE Int. Symp. Inform. Theory, Vail, CO, USA, Jun. 17-22, 2018, pp. 2644-2648.

[7] R. D. Yates, "Age of information in a network of preemptive servers," in Proc. IEEE Int. Conf. on Computer Commun. (INFOCOM), Honolulu, HI, USA, Apr. 15-19 2018, pp. 118-123.

[8] A. Javani, M. Zorgui, and Z. Wang, "Age of information in multiple sensing," in Proc. IEEE Global Telecommun. Conf., Waikoloa, HI, USA, Dec. 9-13, 2019.

[9] S. Farazi, A. G. Klein, and D. Richard Brown, "Average age of information in multi-source self-preemptive status update systems with packet delivery errors," in Proc. Annual Asilomar Conf. Signals, Syst., Comp., Pacific Grove, CA, USA, 2019.

[10] M. Moltafet, M. Leinonen, and M. Codreanu, "Average AoI in multisource systems with source-aware packet management," IEEE Trans. Commun., Early Access, 2020.

[11] - "Moment generating function of the AoI in a two-source system with packet management," IEEE Wireless Commun. Lett., Early Access, [Online]: https://arxiv.org/pdf/2009.14439.pdf, 2021.

[12] M. Moltafet, M. Leinonen, and M. Codreanu, "Average age of information in a multi-source $\mathrm{M} / \mathrm{M} / 1$ queueing model with LCFS prioritized packet management," in Proc. IEEE Int. Conf. on Computer Commun. (INFOCOM) AoI Workshop, Toronto, Canada, Jul. 6-9, 2020, pp. 303308.

[13] _ _ "Average age of information for a multi-source M/M/1 queueing model with packet management," in Proc. IEEE Int. Symp. Inform. Theory, Los Angeles, CA, USA, Jun. 21-26, 2020, pp. 1765-1769.

[14] Q. Kuang, J. Gong, X. Chen, and X. Ma, "Analysis on computationintensive status update in mobile edge computing," IEEE Trans. Veh. Technol., vol. 69, no. 4, pp. 4353-4366, Feb. 2020. 